\title{
Calidad de vida relacionada con la salud, factores psicológicos y fisiopatológicos en pacientes con diagnóstico de Lupus Eritematoso Sistémico - LES
}

\section{Health-related quality of life, psychological and pathophysiological factors in patients with diagnosis of Systemic Lupus Erythematosus - SLE}

\author{
Karem Ariza, Paula Isaza, Ana Milena Gaviria, Japcy Margarita Quiceno, \\ Stefano Vinaccia y Liliana Alvarán \\ Universidad de San Buenaventura, Medellín, Colombia \\ Luis Fernando Pinto, Carlos Jaime Velásquez y Javier Darío Márquez \\ Hospital Pablo Tobón Uribe, Medellín, Colombia
}

(Rec: 10 septiembre 2009 / Acep: 20 abril 2010)

\begin{abstract}
Resumen
El objetivo de este estudio fue analizar, por un lado, los niveles de ansiedad, depresión, apoyo social, índice de actividad lúpica, fibromialgia, daño orgánico y renal, tiempo de diagnóstico de la enfermedad y factores sociodemográficos en 78 pacientes con diagnóstico de Lupus Eritematoso Sistémico; y analizar el efecto de estas variables sobre la calidad de vida relacionada con la salud. Se emplearon como instrumentos: el cuestionario de Salud MOS SF-36, la Escala Hospitalaria de Ansiedad y Depresión HAD, el cuestionario de Apoyo Social Funcional DUKE-UNK, el Índice de Actividad Lúpica SLEDAI y el Índice de Daño Orgánico SLICC-ACR; y se consideró además el diagnóstico médico de Fibromialgia y de Nefropatía. Los resultados señalaron que factores fisiopatológicos (actividad lúpica y daño orgánico), psicológicos (ansiedad-depresión) y sociodemográficos (ocupación y estado civil) y la interacción entre estos afectan la calidad de vida a nivel mental más que físico en los pacientes con lupus.
\end{abstract}

Palabras clave: Lupus Eritematoso Sistémico, calidad de vida relacionada con la salud, ansiedad, depresión, Fibromialgia.

\begin{abstract}
The aim of this research was to evaluate the impact of anxiety, depression, social support, Systemic Lupus Erythematosus (SLE) disease activity index, fibromyalgia, organic and kidney damage, and time of diagnosis of disease on Health-related Quality of Life in 78 patients with SLE diagnosis. Participants completed the Health Survey Questionnaire (SF-36), the Hospital Anxiety and Depression (HAD) scale, the Duke-UNK Functional Social Support questionnaire, the SLEDAI (SLE Disease Activity Index), and the SLICC/ACR damage index for SLE. As a complementary measure, Fibromyalgia and Nephropathy diagnosis was considered. The results shown that in patients with lupus, pathophysiological (SLE disease activity index and organic damage), psychological (anxiety-depression) and sociodemographic factors (occupation and civil status) affect the quality of life in the mental domain rather than in the physical level.
\end{abstract}

Keywords: Systemic Lupus Erythematosus, Health-related Quality of Life, anxiety, depression, fibromyalgia. 


\section{Introducción}

El Lupus Eritematosos Sistémico (LES) es una enfermedad inflamatoria crónica de causa desconocida, aunque la herencia, el entorno y los cambios hormonales juegan un papel importante que afecta a todas las edades, pero con mayor frecuencia a adultos entre los 18 y 50 años con predominio del sexo femenino en una proporción de un hombre por cada 10 a 12 mujeres. La prevalencia del LES varía en los distintos grupos de población, oscilando entre 300 y 400 pacientes por cada 100.000 habitantes. Es más común en ciertos grupos étnicos, especialmente los afroamericanos. Alrededor del $70 \%$ de las personas que sufren lupus presentan la forma sistémica o LES (Molina, Anaya y Molina, 2005).

El LES es un desorden del sistema inmunitario, el cual, normalmente, funciona protegiendo el organismo contra infecciones y virus. En el LES, dicho sistema inmunitario es hiperactivo y se producen importantes cantidades de anticuerpos anormales que reaccionan con los tejidos del propio paciente, afecta a todos los órganos y sistemas, primordialmente las articulaciones, músculos y piel, pero también aunque en menor grado a estructuras internas como los pulmones, corazón, riñones y cerebro (Camargo et al., 2005). La inflamación se refiere a una reacción que provoca dolor, calor, enrojecimiento e hinchazón. En casi la mitad de las personas con LES se ven afectados los órganos internos principales, especialmente los riñones. En la otra mitad de los pacientes con LES están afectadas principalmente la piel y las articulaciones (Gómez et al., 2005).

\section{Calidad de vida relacionada con la salud en pacientes con Lupus Eritematoso Sistémico}

Aparte de los factores fisiológicos antes mencionados, el LES puede afectar de diversas formas la Calidad de Vida Relacionada con la Salud (CVRS) de quienes padecen esta enfermedad, más concretamente a nivel de la actividad física, sexual, mental y social. Desde el punto de vista subjetivo, la calidad de vida relacionada con la salud es la valoración que realiza una persona, de acuerdo con sus propios criterios del estado físico, emocional y social en que se encuentra en un momento dado, y refleja el grado de satisfacción con una situación personal a nivel: fisiológico (sintomatología general, discapacidad funcional, situación analítica, sueño, respuesta sexual), emocional (sentimientos de tristeza, miedo, inseguridad, frustración), y social (situación laboral o escolar, interacciones sociales en general, relaciones familiares, amistades, nivel económico, participación en la comunidad, actividades de ocio, entre otras) (Schwartzmann, 2003). La percepción de CVRS en pacientes con enfermedades crónicas como el LES y las enfermedades reumáticas en edades más tempranas como la niñez y la adolescencia puede vulnerar de manera significativa el desarrollo psicoevolutivo, afectando la autoimagen y los procesos de socialización, de adaptación y más adelante la vida laboral y profesional (Moorthy, Peterson, Harrison, Onel y Lehman, 2007). Por consiguiente, en pacientes adultos jóvenes la CVRS es percibida más negativa que incluso otras enfermedades crónicas como hipertensión, diabetes, infarto de miocardio y paro cardíaco congestivo (CHF) (Meenakshi, 2005). En relación con los síntomas psicológicos que tienen mayor prevalencia en pacientes con LES son: la disfunción cognoscitiva leve como dificultades en la atención y concentración, alteraciones en la memoria verbal y no verbal a largo plazo y compromiso de la memoria viso-espacial a largo plazo; el delirio, la ansiedad, los trastornos del estado de ánimo y la psicosis (Gómez, et al., 2005; Stojanovich, Zandman-Goddard, Pavlovich y Sikanich, 2007). Otros autores como Kuriya, Gladman, Ibáñez y Urowitz (2008) plantean que la CVRS viene afectada también por la comorbilidad del LES con la fibromialgia.

\section{Instrumentos para evaluar calidad de vida en pacientes con Lupus Eritematoso Sistémico}

La calidad de vida relacionada con la salud en pacientes con LES se ha venido evaluando a través de instrumentos tanto genéricos como específicos para enfermedades reumáticas y específicos para el mismo LES (Tabla 1). Según Thumboo y Strand (2007) en una revisión de literatura sobre instrumentos de CVRS utilizados en estudios con pacientes con LES se ha encontrado que las áreas de la CVRS más afectadas en estos pacientes han sido la percepción de salud, la fatiga, el dolor corporal, la actividad funcional a nivel laboral, la autonomía, las relaciones sociales y familiares y la desesperanza aprendida (respecto a la imprevisibilidad de la enfermedad del LES).

\section{Estudios empíricos de calidad de vida en pacientes con Lupus Eritematoso Sistémico}

A continuación se describen a nivel cronológico algunos estudios empíricos y las conclusiones a las que han llegado estos sobre la calidad de vida relacionada con la salud en pacientes con diagnósticos de LES:

Gladman, Urowitz, Gough y MacKinnon (1997) desarrollaron un estudio en Canadá con el objetivo de determinar si en una muestra de 119 pacientes con diagnóstico de LES, el índice de actividad de lúpica, el índice de daño orgánico y la fibromialgia tenían relación con la CVRS. Se utilizaron como instrumentos el Índice de Actividad de Lúpica SLEDAI, el Índice de Daño Orgánico SLICC/ACR y el cuestionario de Salud MOS SF-36, además se evaluaron clínicamente los puntos sensibles de fibromialgia. Se encontró que la presencia de fibromialgia en $21 \%$ de los pacientes no tuvo relación con ninguno de los componentes del SLEDAI ni con el SLICC/ACR, pero sí hubo relación con las ocho dimensiones del cuestionario de Salud MOS 
Tabla 1. Cuestionarios de calidad de vida relacionada con la salud utilizados en estudios con LES

\begin{tabular}{ll}
\hline $\begin{array}{l}\text { Cuestionarios } \\
\text { Generales } \\
\text { de Calidad } \\
\text { de Vida }\end{array}$ & $\begin{array}{l}\text {-Cuestionario de Salud MOS SF-36 (Alonso, Prieto y Antó, 1995a). } \\
\text {-World Health Organization Quality of Life Questionnaire, WHOQOL Group (Lucas, 1998). } \\
\text {-Perfil de Salud de Nottingham, NHP (Alonso, Antó y Moreno, 1995b). } \\
\text {-Perfil de las consecuencias de la Enfermedad, SIP (Badía y Alonso, 1994). }\end{array}$ \\
$\begin{array}{ll}\text { Cuestionarios } \\
\text { Específicos para }\end{array}$ & - Systemic Lupus Erythematosus Quality of Life Questionnaire, SLEQOL (Leong, et al., 2005) \\
LES & -The LupusQoL (McElhone, et al., 2007). \\
\hline $\begin{array}{l}\text { Cuestionarios } \\
\text { Específicos para } \\
\text { enfermedad }\end{array}$ & -The Systemic Lupus Erythamatosus Quality of Life Scale, L-QoL (Doward, et al., 2009) \\
Reumática & -Cuestionario de Impacto de Artritis Reumatoide, AIMS (Abello-Banfi, Cardiel, Ruiz-Mercado y Alarcón-Segovia, \\
Tabla adaptada y modificada de Panopalis y Clarke (2006)
\end{tabular}

SF-36. En conclusión se planteó que la fibromialgia tuvo mayor impacto sobre la CVRS que la misma actividad lúpica y el daño orgánico.

Bruce, Mak, Hallett, Gladman y Urowitz (1999) desarrollaron una investigación en Canadá para evaluar la relación entre la fatiga, la actividad lúpica y el impacto de la enfermedad y la CVRS en pacientes con LES. Se aplicó a una muestra de 81 pacientes con una media de 12,5 años de evolución de la enfermedad el cuestionario de Salud de MOS SF-36, el Índice de Actividad Lúpica SLEDAI y la Medida de Actividad Lúpica Sistémica Revisada SLAM-R, el Índice de Daño Orgánico SLICC/ACR y la escala de Severidad de la Fatiga FSS. No se encontraron correlaciones entre la escala de severidad de fatiga FSS con los índices de actividad lúpica SLEDAI y SLAM-R y con el índice de daño orgánico SLICC/ACR. La fatiga tuvo relación negativa con todas las dimensiones del cuestionario de Salud MOS SF-36. En conclusión, se planteó que la severidad de la fatiga y la comorbilidad con fibromialgia tiene una alta relación con un estado de salud desfavorable en los pacientes con LES.

Dobkin, et al., (1999) en Canadá desarrollaron un estudio con el objetivo de identificar los índices de salud mental y física de la CVRS a una muestra de 129 pacientes con diagnóstico de LES a quienes se les evaluó el distrés psicológico, el apoyo social, el afrontamiento al estrés, la CVRS a través del cuestionario de Salud MOS SF-36, el índice de actividad lúpica mediante el SLAM-R y el índice de daño orgánico con el SLICC-ACR. Los pacientes fueron divididos en dos grupos, los de mayor actividad lúpica $(n=38)$ y menor estado de actividad lúpica $(n=91)$. Los resultados indicaron por un lado, que una mejor salud mental se predecía con un mayor nivel educativo y con menos uso del afrontamiento basado en la emoción en pacientes con más estado de actividad lúpica, y por otro, una mejor salud mental era predecible con menos estrés, menos afrontamiento basado en la emoción y más orientado en la tarea en pacientes durante un estado de menos actividad lúpica. Por otro lado, una mejor salud física se predecía con el uso de un afrontamiento basado en la emoción en los pacientes con un estado más activo de la enfermedad, mientras que una mejor salud física era predecible con menos estrés y menor edad en los pacientes durante un estado menos activo de la enfermedad. En conclusión, la asociación positiva entre el afrontamiento basado en la emoción y una mejor salud física sugiere que durante el estado más activo de la enfermedad pueda ser más útil en situaciones que puedan ser consideradas incontrolables.

Da Costa, et al., (2000) desarrollaron un estudio en Canadá con el propósito de comparar la CVRS en mujeres con diagnóstico de fibromialgia $(n=46)$ y de LES $(n=59)$. Se evaluaron el índice de actividad lúpica en pacientes con LES y los puntos de dolor en pacientes con fibromialgia, también se evaluó estrés diario, afrontamiento al estrés, apoyo social y CVRS con el cuestionario de Salud MOS SF-36. Los resultados mostraron que las dimensiones más afectadas del cuestionario de Salud MOS SF-36 en pacientes con fibromialgia fueron: función física, rol físico, dolor corporal y vitalidad, igualmente que el índice sumario de salud física del MOS SF-36. Se realizaron análisis de regresión jerárquica para determinar los factores relacionados con los índices sumarios de salud mental y física del cuestionario de Salud MOS SF-36 en cada grupo de pacientes, las variables consideradas fueron: edad, ingresos, actividad lúpica (paso 1), estrés diario (paso 2), y apoyo social y afrontamiento basado en la emoción y en la tarea (paso 3). Una mejor salud física en las pacientes con fibromialgia fue relacionada con mayores ingresos, mientras en el grupo de pacientes con LES fue asociada con menos edad, menos actividad lúpica y menos estrés diario. Por otro lado, una salud mental desfavorable en pacientes con fibromialgia fue asociada con más estrés diario, mayor afrontamiento basado en la emoción y menos satisfacción respecto al apoyo social, mientras que bajos ingresos, mayor estrés diario y mayor afrontamiento 
basado en la emoción fueron asociados a una desfavorable salud mental en pacientes con LES. En conclusión, tanto los pacientes con LES como con fibromialgia tuvieron altamente comprometidas las diferentes dimensiones que componente la CVRS.

Kulczycka, Sysa-Jedrzejowska, Zalewska-Janowska, Miniszewska y Robak (2008) en Polonia realizaron una investigación con 83 pacientes con diagnóstico de LES con el propósito de evaluar las relaciones entre la CVRS medida por el cuestionario de salud MOS SF-36 con variables socioeconómicas. Los resultados indicaron en general una CVRS desfavorable en los pacientes con LES. Más concretamente, la duración de la enfermedad y la edad tuvieron una influencia directa sobre la CVRS. Por otro lado, los pacientes que vivían en zonas rurales tuvieron niveles más bajos de CVRS en comparación de quienes vivían en zonas urbanas. Además, los pacientes con mejor nivel educativo evaluaron la percepción de su CVRS más favorable. Los pacientes que describieron como más desfavorables sus condiciones sociales presentaron una mejor percepción de su CVRS. En conclusión, las relaciones entre la CVRS y los factores socioeconómicos fueron relevantes en este estudio.

Mok, Ho, Cheung, Yu y To (2009) desarrollaron en China un estudio con el objetivo de evaluar el efecto de la actividad lúpica y el nivel de daño orgánico sobre la CVRS en 155 pacientes con diagnóstico de LES, a quienes se les aplicó el cuestionario de Salud MOS SF-36, el Índice de Actividad Lúpica SLEDAI y el Índice de Daño Orgánico SLICC-ACR. Los pacientes fueron evaluados por un periodo de dos años. El análisis de regresión reveló que un aumento del índice de daño orgánico era el único factor determinante para la reducción de los índices mental y físico de la CVRS. Los pacientes con una actividad lúpica mayor tuvieron una percepción de dolor corporal y de salud general más desfavorable. Conclusión, la actividad lúpica persistente es asociada con el deterioro en diferentes dimensiones del cuestionario de Salud MOS SF-36 independientemente de la edad, el género, la educación y el estatus socio-económico.

Zheng, et al., (2009) desarrollaron un estudio en China cuyo objetivo fue evaluar la asociación entre CVRS y el apoyo social en pacientes con LES ( $\mathrm{n}=202)$ y compararlo con un grupo control de personas sanas $(n=207)$ y evaluar la influencia de la edad, el índice de actividad de lúpica SLEDAI y la duración de la enfermedad sobre la CVRS. Se aplicaron los cuestionarios de Salud MOS SF-36 y la Escala de Medida de Apoyo Social SSRS. Los resultados evidenciaron que los índices de salud mental y física del cuestionario de Salud MOS SF-36 eran inferiores en la muestra de pacientes con LES en comparación con el grupo control de personas sanas. Los pacientes con LES tuvieron un apoyo social inferior respecto al grupo control en relación con la percepción de apoyo objetivo, subjetivo y la disponibilidad de apoyo. Hubo relación positiva entre el apoyo social y los índices de salud mental y física del cuestionario de Salud MOS SF-36. Además, los índices de salud mental y física del cuestionario de Salud MOS SF-36 se relacionaron negativamente con la edad y el índice de actividad de lúpica. El índice de salud mental del cuestionario de Salud MOS SF-36 se relacionó negativamente con la duración de la enfermedad. En conclusión, se planteó que los pacientes con LES tienen una CVRS y apoyo social desfavorable, y la CVRS puede verse afectada por el apoyo social, la edad y el y índice de actividad lúpica.

La mayoría de los estudios son concluyentes en plantear que es común en pacientes con LES la prevalencia de fibromialgia, y aunque la enfermedad del LES y la fibromialgia son patologías similares "aparentemente", en realidad son trastornos muy distintos (Buskila, Press y Abu-Shakra, 2003). A diferencia del LES, la fibromialgia no es un trastorno autoinmune, no causa inflamación ni interfiere en las funciones de los órganos, sin embargo, muchos de sus síntomas son similares (Staud, 2006). En la fibromialgia igual que en el LES los síntomas son recidivantes, vienen y van, están asociados con fatiga extrema, dolor corporal y desórdenes en la circulación, de hecho hasta el $30 \%$ de las personas con LES desarrollan la enfermedad de fibromialgia después que han sido diagnosticados del mismo LES. Sin embargo no es común que a personas diagnosticadas con fibromialgia se les diagnostique posteriormente la enfermedad del LES. En definitiva, cuando en los pacientes con diagnóstico de LES coexisten ambas patologías (LES y fibromialgia) se ve afectada de manera desfavorable su CVRS (Buskila y Sarzi-Puttini, 2008; Gladman, et al., 1997).

Por lo anterior, el objetivo de este estudio exploratorio ex post-facto transversal con una metodología tipo encuesta fue: 1. Analizar los niveles de los factores psicológicos (ansiedad, depresión y apoyo social), fisiopatológicos (índice de actividad lúpica, presencia de fibromialgia y de daño orgánico y renal, y tiempo de diagnóstico). 2. Analizar el efecto de los factores psicológicos, fisiopatológicos y sociodemográficos sobre la calidad de vida relacionada con la salud en pacientes con diagnóstico de Lupus Eritematoso Sistémico.

\section{Método}

\section{Participantes}

Fueron seleccionados mediante un muestreo no aleatorio (sujetos disponibles) 78 pacientes, 71 mujeres (91\%) y 7 hombres $(9 \%)$ con una edad media de 36,32 años (DT = 11,46) con diagnóstico de LES en tratamiento médico según los criterios del Colegio Americano de Reumatología (Hochberg, 1997) en la Unidad de Reumatología del Hospital Pablo Tobón Uribe (HPTU) de la ciudad de Medellín, Colombia. En cuanto al nivel de escolaridad, un 
$61,6 \%$ realizaron estudios de educación básica (primaria y secundaria), un $24,4 \%$ desarrollaron estudios técnicos y un $14,1 \%$ cursaron estudios universitarios. Respecto al estado civil, un $44,9 \%$ de los participantes eran casados y un $42,3 \%$ solteros, mientras que el resto se encontraron en unión libre $(6,4 \%)$, divorciados $(3,8 \%)$ y viudos $(2,6 \%)$. De acuerdo con la variable ocupación un $42,3 \%$ de los participantes se agruparon en la categoría de amas de casa seguidos de un $32,1 \%$ que se ubicaron en la categoría de empleados, un $9,0 \%$ en desempleados, un $7,7 \%$ eran estudiantes, un $5,1 \%$ eran trabajadores independientes y un $3,8 \%$ se agruparon en la categoría de pensionado/jubilado. En relación con el estrato socioeconómico colombiano un $60,3 \%$ de la muestra eran de un estrato medio (nivel 3 y 4 ) y un 35,9\% eran de un estrato bajo (nivel 1 y 2 ), mientras que el resto $3,8 \%$ pertenecían al estrato alto (nivel 5 y 6), ubicándose la gran mayoría de los participantes en un estrato medio-bajo.

\section{Instrumentos}

La calidad de vida relacionada con la salud se evaluó mediante el Cuestionario de Salud MOS SF-36 en la versión 1 en español (Alonso, et al., 1995a; Alonso, et al., 1998). Este cuestionario mide conceptos genéricos de salud relevantes a través de la edad, la enfermedad y grupos de tratamiento en términos del funcionamiento físico y psicológico, sus ítems detectan tanto estados positivos como negativos de salud (Ware y Sherbourne, 1992). El cuestionario de Salud MOS SF-36 comprende las siguientes ocho dimensiones: función física (limitaciones físicas), rol físico (interferencia en el trabajo y actividades diarias), dolor corporal (intensidad del dolor y su efecto en las actividades), salud general (valoración personal de la salud), vitalidad (sentimiento de energía), función social (interferencia en la vida social habitual), rol emocional (interferencia en el trabajo u otras actividades diarias) y salud mental (depresión, ansiedad, control emocional y de la conducta). El rango de este cuestionario oscila entre 0 a 100 puntos y los puntajes más altos indican una mejor calidad de vida relacionada con la salud.

Para evaluar el nivel de ansiedad y depresión se utilizó la Escala Hospitalaria de Ansiedad y Depresión (HAD), en la versión en castellano desarrollada en Colombia por Rico, Restrepo y Molina (2005). El coeficiente alfa de Cronbach encontrado en la validación fue de 0,85 . El HAD utiliza una escala tipo Likert con 4 posibilidades de respuesta que van de 0 hasta 3 para evaluar de manera situacional la ansiedad y la depresión, con relación al periodo actual. La puntuación total en cada subescala de 7 preguntas se obtiene sumando los ítems correspondientes, en un rango de 0 a 21 , tanto para la puntuación de ansiedad como de depresión se considera de 0 a 7 normal, de 8 a 10 dudoso y de 11 o más problema clínico. A mayor puntuación, más altos niveles de ansiedad o depresión.
El apoyo social fue medido por el cuestionario de Apoyo Social Funcional DUKE-UNK en la versión española De la Revilla, Baylon y De Dios (1991). Este cuestionario de auto evaluación está formado por 11 ítems que recogen la opinión del individuo sobre la disponibilidad de las personas que le ofrecen ayuda ante las dificultades, facilidades para la relación social y para comunicarse de forma empática y emotiva. Estos aspectos se evalúan mediante una escala likert con 5 opciones de respuesta que se puntúan de 1 (mucho menos de lo que deseo) hasta 5 (tanto como deseo). El punto de corte establecido por los autores para considerar percepción de apoyo social fue de 32 puntos en adelante. El alfa de cronbach de la validación española en diferentes investigaciones tuvo puntajes que oscilaron entre 0,80 y 0,90 .

El índice de actividad lúpica se evaluó mediante el SLEDAI (Systemic Lupus Erythematosus Disease Activity Index) desarrollado por Gladman, Ibañez y Urowitz (2002). Esta escala que sirve para cuantificar la actividad de la enfermedad en relación con variables clínicas y de laboratorio. El índice comprende 24 ítems agrupadas en 4 tópicos que incluyen 8 manifestaciones neurológicas, 8 vasculares, 4 músculo- esqueléticas, 4 renales, 2 hematológicas, 2 cutáneas, 2 serosas, 2 inmunológicas y 1 manifestación sistémica. Cada ítem se puntúa considerando la presencia de actividad lúpica en el momento de la evaluación o en su defecto en los últimos 10 días antes de esta. El rango de esta escala oscila entre 0 a 105 puntos y la actividad lúpica de la enfermedad se clasifica de la siguiente manera: Inactividad (0 puntos), Leve (1-5 puntos), Moderada (6-10 puntos), Alta (11-20 puntos) y Muy alta ( $>20$ puntos).

El índice de daño orgánico se evaluó mediante el Systemic Lupus International Collaborating Clinics y el American College of Rheumatology (SLICC-ACR) desarrollado por Gladman, et al., (1996) que sirve para determinar el daño orgánico del LES independientemente de la actividad de la misma, ya que por si sola no explicaría la morbimortalidad de esta enfermedad. El índice esta compuesto por 39 ítems agrupados en doce dimensiones. Cada ítem se puntúa considerando si hay presencia o ausencia de este con un tiempo de referencia de por lo menos seis meses de presencia previos a la evaluación para considerársele positivo y/o descartar que se deba solamente a la actividad de la enfermedad. El rango de los puntajes oscilan entre 0 (ausencia de daño orgánico) hasta 47 (dañó orgánico severo), a partir de un puntaje de 1 se puede considerar daño orgánico y a mayor puntaje el pronóstico es más desfavorable.

Para el diagnóstico de fibromialgia en los pacientes con LES se tuvo en cuenta los criterios de Croft, Schollum y Silman (1994) para determinar el dolor difuso de tres meses o más de duración que afecta al esqueleto axial y al menos a dos cuadrantes contralaterales del cuerpo y dolor 
en al menos en 11 de 18 localizaciones denominadas puntos gatillo cuando se palpan con la presión suficiente como para palidecer el lecho ungueal.

\section{Procedimiento}

Previa autorización de la dirección científica de la Unidad de Reumatología del Hospital Pablo Tobón Uribe (HPTU) de la ciudad de Medellín, Colombia, y acceso a las bases de datos se contactaron a los pacientes con diagnóstico de LES para informarles sobre los propósitos de la investigación. Luego de la lectura y firma de la carta de consentimiento informado por parte de los pacientes con LES se aplicaron con la ayuda y supervisión de los psicólogos la ficha de datos sociodemográficos y tres pruebas psicológicas (MOS SF-36, HAD y DUKE-UNK). Mientras que las pruebas fisiopatológicas como el SLEDAI, el SLICC-ACR, la medida clínica sobre los puntos gatillo de fibromialgia fueron aplicadas por los médicos especialistas en reumatología de acuerdo con los reportes de los pacientes con LES, y los resultados de nefropatología lúpica (daño renal) fueron obtenidos a través de exámenes de laboratorio clínico.

\section{Resultados}

A continuación se presentan los resultados descriptivos del estudio, la media, la desviación típica (DT) y los valores mínimos y máximos, y el índice de consistencia interna coeficiente Alfa de Cronbach de cada una de las variables psicológicas (Tabla 2); mientras que las variables categóricas o nominales se muestran a nivel de porcentajes (Tabla 3).

\section{Factores Psicológicos}

A nivel general se observa índices muy altos de consistencia interna en cada una de las dimensiones y subescalas de los cuestionarios psicométricos utilizados en el estudio.

Se comparan en la tabla 3 (sólo a manera de información y clarificación de los datos) nuestros resultados con los obtenidos en población sana Colombiana de la ciudad de Medellín con el cuestionario de salud MOS SF-36 (García y Lugo, 2002). Encontrando que las dimensiones de calidad de vida son un poco más desfavorables en los pacientes con LES.

En relación a las puntuaciones medias de la escala hospitalaria de ansiedad y depresión (HAD), se puede apreciar en sus subescalas puntajes bajos respecto a los puntos de corte de 8 para la subescala de Ansiedad y 9 para la subescala de Depresión planteados por Rico, Restrepo y Molina (2005) para indicar si los pacientes tienen síntomas o no de ansiedad y depresión en Colombia. En cuanto al apoyo social (DUKE-UNK) se tuvo valores medios muy altos en comparación con los puntos de corte (32 puntos) planteados por De la Revilla, et al., (1991) para considerar la presencia de un buen apoyo social.

\section{Factores Fisiopatológicos}

A nivel general se observa en la tabla 4 que la gran mayoría de los pacientes con LES tenían un tiempo de diagnóstico de la enfermedad superior entre los cinco y diez años, quienes prácticamente no presentaron comorbilidad con el diagnóstico de fibromialgia, con una actividad lúpica que oscila entre niveles de ausentes a moderados, con presencia moderada de daño orgánico y con muy baja presencia de daño renal.

Tabla 2. Estadísticas descriptivas y Alfa de Cronbach de los factores psicológicos.

\begin{tabular}{|c|c|c|c|c|c|}
\hline & Media & DT & Mínimo & Máximo & Alfa \\
\hline \multicolumn{6}{|l|}{ Cuestionario MOS SF-36: } \\
\hline Función Física (FF) & 65,32 & 28,02 & 0 & 100 & 0,85 \\
\hline Rol Físico (RF) & 54,49 & 45,89 & 0 & 100 & 0,85 \\
\hline Dolor Corporal (DC) & 56,74 & 29,81 & 0 & 100 & 0,85 \\
\hline Vitalidad (VT) & 58,46 & 25,92 & 0 & 100 & 0,85 \\
\hline Salud General (SG) & 57,37 & 24,38 & 5 & 100 & 0,85 \\
\hline Función Social (FS) & 70,55 & 28,49 & 0 & 100 & 0,84 \\
\hline Salud Mental (SM) & 66,82 & 26,95 & 0 & 100 & 0,85 \\
\hline Rol Emocional (RE) & 64,37 & 43,46 & 0 & 100 & 0,87 \\
\hline \multicolumn{6}{|l|}{ Escala HAD: } \\
\hline HAD Ansiedad & 7,12 & 4,80 & 0 & 19 & 0,89 \\
\hline HAD Depresión & 4,71 & 4,38 & 0 & 19 & 0,89 \\
\hline Cuestionario de Apoyo Social DUKE-UNK: & 46,29 & 8,47 & 21 & 55 & 0,86 \\
\hline
\end{tabular}


DiAgNóstico DE Lupus ERITEMATOSO SistÉMICO -LES-

Tabla 3. Comparación entre las medias de las dimensiones del cuestionario de Salud MOS SF-36 entre una muestra sana y pacientes de LES.

\begin{tabular}{|c|c|c|c|c|c|c|c|c|}
\hline MUESTRA & $\begin{array}{c}\text { Función } \\
\text { Física } \\
(\mathrm{FF})\end{array}$ & $\begin{array}{c}\text { Rol } \\
\text { Físico } \\
(\mathrm{RF}) \\
\end{array}$ & $\begin{array}{c}\text { Dolor } \\
\text { Corporal } \\
(\mathrm{DC})\end{array}$ & $\begin{array}{c}\text { Salud } \\
\text { General } \\
(\mathrm{SG})\end{array}$ & $\begin{array}{l}\text { Vitalidad } \\
\text { (VT) }\end{array}$ & $\begin{array}{c}\text { Función } \\
\text { Social } \\
(\mathrm{FS})\end{array}$ & $\begin{array}{c}\text { Rol } \\
\text { Emocional } \\
(\mathrm{RE})\end{array}$ & $\begin{array}{c}\text { Salud } \\
\text { Mental } \\
(\mathrm{SM}) \\
\end{array}$ \\
\hline Personas sanas & 93,9 & 90 & 82,0 & 84,4 & 75,8 & 85,8 & 86,3 & 79,5 \\
\hline Pacientes de LES & 65,3 & 54,4 & 56,7 & 57,3 & 58,4 & 70,5 & 64,3 & 66,8 \\
\hline
\end{tabular}

Tabla 4. Características de los factores fisiopatológicos.

\begin{tabular}{lll}
\hline & & Porcentaje (\%) \\
\hline Tiempo de Diagnóstico & Menos de 1 año & $9(11,5 \%)$ \\
& Entre 1 y 5 años & $19(24,4 \%)$ \\
& Entre 5 y 10 años & $27(34,6 \%)$ \\
& Más de 10 años & $23(29,5 \%)$ \\
\hline Fibromialgia & Si & $2(2,6 \%)$ \\
& No & $76(97,4 \%)$ \\
\hline Actividad Lúpica SLEDAI & Ausencia & $36(46,2 \%)$ \\
& Leve & $14(17,9 \%)$ \\
& Moderada & $17(21,8 \%)$ \\
\hline Daño Orgánico SLICC-ACR & Alta & $6(7,7 \%)$ \\
& Muy Alta & $5(6,4 \%)$ \\
\hline Daño Renal & Negativo & $49(62,8 \%)$ \\
& Positivo & $29(37,2 \%)$ \\
\hline
\end{tabular}

Por otro lado, de acuerdo a los resultados del análisis de la varianza factorial, que se realizaron con el fin de conocer el efecto individual y el efecto de las interacciones que las variables independientes (sociodemográficas, psicológicas y fisiopatológicas) pudieron tener sobre la calidad de vida relacionada con la salud de los pacientes con LES, controlando el posible efecto modificador de la edad a través de un análisis de covarianza - ANCOVA se encontró que: la interacción de las variables depresión y daño orgánico tuvieron un efecto significativo sobre la dimensión función social $\left(R^{2}=0,64 \% ; p=0,043\right)$. De igual modo la ansiedad la tuvo pero sobre la dimensión vitalidad $\left(R^{2}=56,2 \% ; p=0,028\right)$ y la característica sociodemográfica estado civil con la salud general $\left(R^{2}=78,4 \% ; p=0,025\right)$. En cuanto la depresión ( $p=0,022)$, la ocupación $(p=0,036)$ y la interacción entre ansiedad-actividad lúpica $(p=0,033)$ tuvieron un efecto estadísticamente significativo sobre la dimensión rol físico $\left(R^{2}=79,7 \%\right)$. De igual modo se halló que las variables ansiedad $(p=0,000)$, depresión $(p=0,006)$ y la interacción ocupación-actividad lúpica $(p=0,028)$ tuvieron un efecto significativo sobre la dimensión salud mental $\left(R^{2}=86,4 \%\right)$.

\section{Discusión}

La mayoría de los pacientes con LES de este estudio fueron mujeres adultas jóvenes, amas de casa, con un nivel de estudios básicos (primaria y secundaria) y de estrato socioeconómico colombiano medio-bajo.

Respecto a los factores psicológicos (calidad de vida, ansiedad-depresión y apoyo social) a nivel general se encontraron muy buenos niveles en los pacientes con LES de este estudio. La calidad de vida relacionada con la salud fue favorable, siendo las dimensiones asociadas con la salud mental mucho mejores que las asociadas a la salud física. De acuerdo con la dimensión Función Física (FF) que tuvo los niveles más altos dentro de las dimensiones de salud física del cuestionario de Salud MOS SF-36, se observa que los pacientes de LES tuvieron muy pocas dificultades que limitaran sus actividades físicas y de auto cuidado que implicaran esfuerzos moderados e intensos. Respecto a rol físico, se observa que la enfermedad no interfiere en las actividades relacionadas con el rol diario (trabajo, estudio, etc.). En cuanto a la dimensión dolor corporal se aprecia que los pacientes no tienen una gran interferencia del dolor en sus actividades de trabajo y de hogar. Igualmente en la dimensión de salud general se percibe una valoración positiva sobre la salud actual, las perspectivas de salud en el futuro y la resistencia a enfermarse. Por otro lado, se evidencia además, altos sentimientos de energía y vitalidad frente al sentimiento de cansancio y agotamiento. En cuanto a rol emocional, se observa que en estas personas no se presentaron interferencias significativas de lo emocional en las actividades diarias, favoreciéndoles tener el rendimiento adecuado en el hogar y el trabajo. Consecuentemente, no se 
evidenció significativamente niveles emocionales negativos (ansiedad-depresión-estrés) en cuanto a la dimensión de salud mental, posibilitándoles a los pacientes del estudio poder tener un buen control emocional en sus vidas. Lo anterior se corrobora con los resultados de la escala HAD donde se encontró que la sintomatología ansioso-depresiva era ausente. Respecto a la dimensión función social es de resaltar que es una de las más altas en relación con todas las dimensiones en general del cuestionario de salud MOS SF-36, observándose mínimos problemas de salud física $\mathrm{y} / \mathrm{o}$ emocional que interfieran en la vida social habitual de estos pacientes. Es de anotar además la alta percepción de Apoyo Social (DUKE-UNK) que se encontró en el estudio, indicando que los pacientes con LES no presentan dificultades para relacionarse y tienen posibilidades de comunicarse de forma empática y emotiva con los demás tanto a nivel confidencial como afectivo.

Los estudios de CVRS en muestras de pacientes con LES no han sido concluyentes hasta el momento, ya que unos plantean, por un lado, que el LES afecta significativamente a nivel físico y mental la CVRS, mientras que otros argumentan que la afectación es más leve. Al respecto, en este estudio la mayoría de los pacientes con LES tuvieron una CVRS muy favorable igual que en investigaciones desarrolladas en la ciudad de Medellín, Colombia, con pacientes con LES donde se ha encontrado en todas las dimensiones que conforman el cuestionario de Salud MOS SF-36 muy buen nivel de bienestar físico y mental en general (HincapiéZapata, Suárez-Escudero, Pineda-Tamayo y Anaya, 2009; Vinaccia, et al., 2006). Mientras por el contrario, otros estudios han hallado una CVRS más desfavorable en pacientes con LES en comparación con muestras de control sanas y aún más con otras enfermedades crónicas (Abu-Shakra, et. al., 1999; Alarcón, et al., 2004; Jolly, 2005; Kulczycka, et al., 2008; Lash, 1998; Meenakshi, 2005; Rinaldi, et al., 2001; Rinaldi, et al., 2004).

En cuanto a la presencia de síntomas de ansiedad y depresión en pacientes con LES y enfermedades crónicas en general la mayoría de los estudios argumentan que es alta la prevalencia de estos (Kozora, Ellison y West, 2006; Meenakshi, 2005; Serrano, 2002), se ha encontrado además, que la depresión afecta la salud mental y física de la CVRS y la ansiedad afecta la salud mental de CVRS, mientras que en otros estudios ambas afectan la CVRS de los pacientes con LES (Doria, et. al., 2004; Meenakshi, 2005; Sharpe, Denton y Schrieber, 2004; Tam, et al., 2008; Thumboo y Strand, 2007; Wang, Mayo y Fortin, 2001). Por el contario, en un estudio desarrollado en la ciudad de Medellín, Colombia con pacientes con LES no se encontraron niveles clínicamente significativos de ansiedad-depresión que afectarán la CVRS en general, más sin embargo la depresión sí fue una variable predictiva negativa de la dimensión función social del cuestionario de Salud MOS SF-36 (Vinaccia, et al., 2006). Resultados que son muy similares a nuestro estudio, donde los síntomas de ansiedad-depresión no fueron elevados pero sí afectaron algunas dimensiones del cuestionario de Salud MOS SF-36, donde se encontró que la depresión con daño orgánico interfiere en la función social, la ansiedad afecta la vitalidad y la ansiedad y la depresión impactan de manera significativa sobre la salud mental, mientras que por otro lado, la depresión interfiere sobre el rol físico de los pacientes con LES.

De acuerdo con los factores fisiopatológicos como tiempo de diagnóstico de la enfermedad, actividad lúpica, diagnóstico asociado con fibromialgia, y daño orgánico y renal se encontraron frecuencias muy bajas. El tiempo de diagnóstico en los pacientes con LES fue superior a cinco años y en cuanto a la actividad lúpica que hace referencia a las manifestaciones neurológicas, vasculares, músculo esqueléticas, renales, hematológicas, cutáneas, cerosas, inmunológicas y sistémicas se halló que oscilaba entre ausente a moderada. La comorbilidad del LES con la fibromialgia fue prácticamente ausente, de igual modo la presencia de daño renal, mientras que la presencia de daño orgánico fue moderada.

Al respecto, los estudios no son concluyentes respecto al tiempo de diagnóstico de la enfermedad de LES, ya que hay investigaciones que demuestran que no hay cambios significativos en la CVRS en pacientes con LES evaluados durante ocho años (Kuriya, et al., 2008), mientras que otros autores plantean que los años de evolución de la enfermedad pueden afectar de manera importante la percepción de salud general de la CVRS (Freire, Maia, Nepomuceno y Ciconelli, 2007; Kulczycka, et al., 2008). Se ha visto incluso en otros tipos de enfermedades crónicas, véase hipertensión, diabetes, asma y migraña que la CVRS puede fluctuar a través del tiempo (Bazán, Osorio, Paredes y Ríos, 2003). En nuestro estudio por el contrario el tiempo de diagnóstico no fue una variable estadísticamente significativa.

De acuerdo con la actividad lúpica, muchos estudios son concluyentes en que es un factor determinante para la percepción favorable o no de la CVRS, incluso independientemente de la edad, el género, la educación y el estatus socio-económico (Da Costa, et al., 2000; Dobkin, et al., 1999; Freire, et al., 2007; Mok, et al., 2009; Wang, et al., 2001; Ruperto, et al., 2004; Zheng, et al., 2009). Mas sin embargo en nuestro estudio se observa que la interacción de la variable sociodemográfica ocupación con la actividad lúpica afecta de manera significativa sobre la dimensión salud mental, mientras que la ansiedad asociada a la actividad lúpica afecta el rol físico de la CVRS.

Por otro lado, muchos estudios son concluyentes en plantear que la comorbilidad del LES con la enfermedad de fibromialgia afecta de manera importante la CVRS a diferencia de tener sólo el diagnóstico de LES (Bruce, et al., 1999; Buskila y Sarzi-Puttini, 2008; Da Costa, et al., 2000; Gladman, et al., 1997; Kuriya, et al., 2008). Esto pondría 
Diagnóstico de Lupus ERITEMATOSO Sistémico -LES-

ser uno de los factores explicativos de la CVRS favorable de los pacientes con LES ya que esta comorbilidad fue mínima en nuestro estudio.

En cuanto al efecto acumulativo de la enfermedad sobre los órganos o toxicidad por medicamentos (daño orgánico) sobre la CVRS los estudios no son concluyentes: en unos no se encuentran relaciones significativas mientras que en otros se ha encontrado que los índices físico y mentales de la CVRS se ven afectados y otros han encontrado que el daño orgánico afecta algunas dimensiones como la función física y la función social del cuestionario de Salud MOS SF-36 (Bruce, et al., 1999; Gladman, et al., 1997; Mok, et al., 2009; Ruperto, et al., 2004; Wang, et al., 2001). Consecuentemente en nuestro estudio se observa que el daño orgánico asociado con síntomas de depresión afecta la función social de la CVRS.

Respecto a la presencia de daño renal algunos estudios argumentan que la CVRS puede reducirse de manera significativa mientras que otros plantean lo contrario, ya que los pacientes pueden en el tiempo tener un proceso de adaptación progresivo a las limitaciones asociadas a la enfermedad de LES (Clarke, et al., 2008; Hanly, McCurdy, Fougere, Douglas y Thompson, 2004; Ruperto, et al., 2004). En nuestro estudio no hubo relación estadísticamente significativa de daño renal sobre la CVRS lo que podría explicarse por la baja presencia de esta variable en el estudio.

Por último, se ha encontrado en la literatura que el impacto de las variables sociodemográficas como edad, género, escolaridad, ocupación, estado civil y estrato socioeconómico sobre la CVRS tienen un peso importante (Alarcón, et al., 2004; Kulczycka, et al., 2008). En nuestro estudio es de anotar que la característica sociodemográfica ocupación tuvo un efecto significativo sobre el rol físico, mientras que el estado civil lo tuvo sobre la salud general de la CVRS.

En conclusión, en este estudio se encontró que los factores fisiopatológicos (actividad lúpica y daño orgánico) psicológicos (ansiedad-depresión) y sociodemográficos (ocupación y estado civil) y la interacción entre estos son los que afectan la calidad de vida a nivel mental más que físico en los pacientes con lupus.

\section{Referencias}

Abello-Banfi, M., Cardiel, M.H., Ruiz-Mercado, R., \& Alarcón-Segovia, D. (1994). Quality of life in rheumatoid arthritis: Validation of a spanish version of the Arthritis Impact Measurement Scales (Spanish-AIMS). Journal of Rheumatology, 21, 50-55.

Abu-Shakra, M., Mader, R., Langevitz, P., Friger, M., Codish, S., Neumann, L., et al. (1999). Quality of life in systemic lupus erythematosus: a controlled study. Journal of Rheumatology, 26, 306-309.

Alarcón, G.S., McGwin, G., Uribe, A., Friedman, A.W., Roseman, J.M., Fessler, B.J., et al. (2004). Systemic lupus erythematosus in a multiethnic lupus cohort (LUMINA). XVII. Predictors of self-reported health-related quality of life early in the disease course. Arthritis \& Rheumatism, 51, 465-474.
Alonso, J., Antó, J.M., \& Moreno, C. (1995b). Spanish version of the Nottingham Health Profile: traslation and preliminary validity. American Journal Public Health, 80, 704-708.

Alonso, J., Prieto, L., \& Antó, J.M. (1995a). La versión española del SF-36 Health Survey (Cuestionario de Salud SF-36): un instrumento para la medida de los resultados clínicos. Medicina Clínica, 104, 771-776.

Alonso, J., Regidor, E., Barrio, G., Prieto, L., Rodríguez, C., y De la Fuente, L. (1998). Valores poblacionales de referencia de la versión española del Cuestionario de Salud SF-36. Medicina Clínica, 111, 410-416.

Badía, X., \& Alonso, J. (1994). Adaptación de una medida de la disfunción relacionada con la enfermedad: la versión española del Sickness Impact Profile. Medicina Clínica, 102, 90-95.

Badía, X., Roset, M., Montserrat, S., Herdman, M., \& Segura, A. (1999). La versión española del EuroQol: descripción y aplicaciones. Medicina Clínica, 112, 79-86.

Bazán, G.E., Osorio, M., Paredes, P., \& Ríos, M.R. (2003). Evaluación de la calidad de vida en pacientes adultos con diferentes enfermedades crónicas. Psicología conductual, 11, 307-318.

Bruce, I.N., Mak, V.C., Hallett, D.C., Gladman, D.D., \& Urowitz, M.B. (1999). Factors associated with fatigue in patients with systemic lupus erythematosus. Annals of the Rheumatic Diseases, 58, 379-381.

Buskila, D., Press, J., \& Abu-Shakra, M. (2003). Fibromyalgia in systemic lupus erythematosus: prevalence and clinical implications. Clinical Reviews in Allergy and Immunology, 25, 25-28.

Buskila, D., \& Sarzi-Puttini, P. (2008). Fibromyalgia and Autoimmune Diseases: the Pain behind Autoimmunity. The Israel Medical Association Journal, 10, 77-78.

Camargo, J.F., Tobón, G.J., Fonseca, N., Díaz, J.L., Uribe, M., Molina, F., et al. (2005). Autoimmune rheumatic diseases in the intensive care unit: experience from a tertiary referral hospital and review of the literature. Lupus, 4, 315-320.

Cardiel, M.H., Abello-Banfi, M., Ruiz-Mercado, R., \& Alarcón-Segovia, D. (1993). How to measure health status in rheumatoid arthritis in non english speaking patients: Validation of the health assesment questionaire disability index (Spanish HAQ-DI). Clinical and Experimental Rheumatology, 11, 117-121.

Clarke, A.E., Panopalis, P., Petri, M., Manzi, S., Isenberg, D.A., Gordon, C., et al. (2008). SLE patients with renal damage incur higher health care costs. Rheumatology (Oxford, England), 47, 329-333.

Croft, P., Schollum, J., \& Silman, A., (1994). Population study of tender point counts and pain as evidence of fibromyalgia. The British Medical Journal, 309, 696-699.

Da Costa, D., Dobkin, P.L., Fitzcharles, M.A., Fortin, P.R., Beaulieu, A., Zummer, M., et al. (2000). Determinants of health status in fibromyalgia: a comparative study with systemic lupus erythematosus. The Journal of Rheumatology, 27, 65-72.

De la Revilla, L., Baylon, E., \& de Dios, J. (1991). Validación de una escala de apoyo social funcional para su uso en la consulta del medico de familia. Atención Primaria, 9, 668.

Dobkin, P.L., Da Costa, D., Dritsa, M., Fortin, P.R., Senécal, J.L., Goulet, J.R., et al. (1999). Quality of life in systemic lupus erythematosus patients during more and less active disease states: differential contributors to mental and physical health. Arthritis Care \& Research, $12,401-410$.

Doria, A., Rinaldi, S., Ermani, M., Salaffi, F., Laccarino, L., Ghirardello, A., et al. (2004). Role of clinical, immunological and psychological determinants. Rheumatology, 51, 989-995.

Doward, L.C., McKenna, S.P., Whalley, D., Tennant, A., Griffiths, B., Emery, P., et al. (2009). The Development of the L-QoL: A quality of life instrument specific to Systemic Lupus Erythematosus. Annals of the Rheumatic Diseases, 68, 196-200.

Freire, E.A., Maia, I.O., Nepomuceno, J.C., \& Ciconelli, R.M. (2007). Damage index assessment and quality of life in systemic lupus erythematosus patients (with long-term disease) in Northeastern Brazil. Clinical Rheumatology, 26, 423-428.

García, H., \& Lugo, H. (2002). Adaptación cultural y fiabilidad del instrumento de calidad de vida SF36 en instituciones de Medellín. Tesis de Maestría. Medellín: facultad nacional de salud pública. 
Gladman, D., Ginzler, E., Goldsmith, C., Fortin, P., Liang, M., Urowitz, M., et al. (1996). The development and initial validation of the systemic lupus international collaborating Clinics/American college of rheumatology damage index for Systemic Lupus. Erythematosus. Arthritis \& Rheumatism, 39, 363-369.

Gladman, D.D., Ibañez, D., \& Urowitz, M.B. (2002). Systemic lupus erythematosus disease activity index 2000. The Journal of Rheumatology, 29, 288-291.

Gladman, D.D., Urowitz, M.B., Gough, J., \& MacKinnon, A. (1997). Fibromyalgia is a major contributor to quality of life in lupus. The Journal of Rheumatology, 24, 2145-2148.

Gómez, L.M., Anaya, J.M., González, C.I., Pineda-Tamayo, R., Otero, W., Arango, A., et al. (2005). Polymorphism in Colombian patients with autoimmune diseases. Genes Immunology, 6, 628-631.

Grootscholten, C., Ligtenberg, G., Derksen, R.H.W.M., Schreurs, K.M.G., Glas-Vos, J.W., Hagen, E.C., et al. (2003). Health-related quality of life in patients with systemic lupus erythematosus: Development and validation of a lupus specific symptom checklist. Quality of Life Research, 12, 635-644.

Hanly, J.G., McCurdy, G., Fougere, L., Douglas, J.A., \& Thompson, K. (2004). Neuropsychiatric events in systemic lupus erythematosus: attribution and clinical significance. British Journal of Rheumatology, 31, 2156-2162.

Hincapié-Zapata, M.E., Suárez-Escudero, J.C., Pineda-Tamayo, R., \& Anaya, J.M. (2009). Calidad de vida en esclerosis múltiple y otras enfermedades crónicas autoinmunes y no autoinmunes. Revista de Neurología, 48, 225-230.

Hochberg, M.C. (1997). Updating the American College of Rheumatology revised criteria for the classification of systemic lupus erythematosus letter. Arthritis \& Rheumatism, 40, 17-25.

Jolly, M. (2005). How does quality of life of patients with systemic lupus erythematosus compare with that of other common chronic illnesses?. Journal Rheumatology, 32, 1706-1708.

Kozora, E., Ellison, M.C., \& West, S. (2006). Depression, fatigue, and pain in systemic lupus erythematosus (SLE): relationship to the American College of Rheumatology SLE neuropsychological battery. Arthritis \& Rheumatism, 55, 628-635.

Kulczycka, L., Sysa-Jedrzejowska, A., Zalewska-Janowska, A., Miniszewska, J., \& Robak, E. (2008). Quality of life and socioeconomic factors in Polish patients with systemic lupus erythematosus. The Journal of the European Academy of Dermatology and Venereology, 22, 1218-1226.

Kuriya, B., Gladman, D.D., Ibañez, D., \& Urowitz, M.B. (2008). Quality of life over time in patients with systemic lupus erythematosus. Arthritis \& Rheumatism, 59, 181-185.

Lash, A.A. (1998). Quality of life in systemic lupus erythematosus. Applied Nursering Research, 11, 130-137.

Leong, K.P., Kong, K.O., Thong, B.Y., Koh, E.T., Lian, T.Y., Teh, C.L., et al. (2005). Development and preliminary validation of a systemic lupus erythematosus-specific quality-of-life instrument (SLEQOL). Rheumatology, 44, 1267-1276.

Lucas, R. (1998). Versión española del WHOQOL. Barcelona, España: Ergón.

Meenakshi, J. (2005). ¿How Does Quality of Life of Patients with Systemic Lupus Erythematosus Compare with That of Other Common Chronic Illnesses? The Journal of Rheumatology, 32, 1706-1708.

McElhone, K., Abbott, J., Shelmerdine, J., Bruce, I.N., Ahmad, Y., Gordon, C., et al. (2007). Development and validation of a disease-specific health-related quality of life measure, the LupusQol, for adults with systemic lupus erythematosus. Arthritis \& Rheumatism, 57, 972-979.
Mok, C.C., Ho, L.Y., Cheung, M.Y., Yu, KL., \& To, C.H. (2009). Effect of disease activity and damage on quality of life in patients with systemic lupus erythematosus: a 2-year prospective study. Scandinavian Journal of Rheumatology, 38 (2), 121-127.

Molina, J.F., Anaya, J.M., \& Molina, J. (2005). Lupus Eritematoso: Manual práctico para médicos y pacientes (2 ${ }^{\mathrm{a}}$. ed.). Medellín, Colombia: CIB.

Moorthy, L.N., Peterson, M.G., Harrison, M.J., Onel, K.B., \& Lehman, T.J. (2007). Quality of life in children with systemic lupus erythematosus: a review. Lupus, 16, 663-669.

Panopalis, P., \& Clarke, A.E. (2006). Quality of life in systemic lupus erythematosus. Clinical \& Developmental Immunology, 13, 321-324.

Rico, J.L., Restrepo, M., \& Molina, M. (2005). Adaptación y validación de la escala hospitalaria de ansiedad y depresión (HAD) en una muestra de pacientes con cáncer del Instituto Nacional de Cancerología de Colombia. Avances en Medición, 3, 73-86.

Rinaldi, S., Doria, A., Salaffi, F., Ermani, M., Laccarino, L., Ghirardello, A., et al. (2004). Health-related quality of life in Italian patients with systemic lupus erythematosus. Relationship between physical and mental dimension and impact of age. Rheumatology, 43, 1574-1579.

Rinaldi, S., Doria, A., Vescovi, F., Corbanese, S., Laccarino, L., Della, S., et al. (2001). Quality of life in systemic lupus erythematosus. Reumatismo, 53, 108-115.

Ruperto, N., Buratti, S., Duarte-Salazar, C., Pistorio, A., Reiff, A., Bernstein, B., et al (2004). Health-related quality of life in juvenile-onset systemic lupus erythematosus and its relationship to disease activity and damage. Arthritis and Rheumatism, 51, 458-464.

Sharpe, L., Denton, F., \& Schrieber, L. (2004). Validity of the disease repercussion profile in patients with systemic lupus erythematosus. Lupus, 13, 428-435.

Schwartzmann, L. (2003). Calidad de vida relacionada con la salud: aspectos conceptuales. Ciencia y Enfermería, 2, 9-21.

Serrano, F. (2002). Depresión en enfermedades médicas. Anales del Sistema Sanitario de Navarra, 25, 137-148.

Staud, R. (2006). Are patients with systemic lupus erythematosus at increased risk for fibromyalgia? Current Rheumatology Reports, 8, 430-435.

Stojanovich, L., Zandman-Goddard, G., Pavlovich, S., \& Sikanich, N. (2007). Psychiatric manifestations in systemic lupus erythematosus. Autoimmunity Reviews, 6, 421-426.

Tam, L.S., Wong, A., Mok, V.C., Zhu, Y.E., Kwok, L.W., Li, T.K., et al. (2008). The relationship between neuropsychiatric, clinical, and laboratory variables and quality of life of Chinese patients with systemic lupus erythematosus. The Journal of Rheumatology, 35, 1038-1045.

Thumboo, J., \& Strand, V. (2007). Health-related quality of life in patients with systemic lupus erythematosus: an update. The Annals, Academy of Medicine, Singapore, 36, 115-122.

Vinaccia, S., Quiceno, J.M., Zapata, C., Abad, L., Pineda, R., \& Anaya, J.M. (2006). Calidad de vida en pacientes con lupus eritematoso sistémico. Revista Colombiana de Psicología, 15, 57-65.

Wang, C., Mayo, N.E., \& Fortin, P.R. (2001). The relationship between health related quality of life and disease activity and damage in systemic lupus erythematosus. British Journal of Rheumatology, 28, 525-532.

Ware, J., \& Sherbourne, C.D. (1992). The MOS 36-item short-form health survey (SF-36). I. Conceptual framework and item selection. Medical Care, 30, 473-483.

Zheng, Y., Ye, D.Q., Pan, H.F., Li, W.X., Li, L.H., Li, J., et al. (2009). Influence of social support on health-related quality of life in patients with systemic lupus erythematosus. Clinical Rheumatology. 28, 265-269. 